\title{
PALEO
}

Revue d'archéologie préhistorique

\section{Le débitage sur enclume aux Bosses (Lamagdelaine, Lot, France)}

Bipolar-on-anvil debitage at Les Bosses (Lamagdelaine, Lot, France)

Vincent Mourre, Marc Jarry, David Colonge et Laure-Amélie Lelouvier

\section{(2) OpenEdition}

1 Journals

Édition électronique

URL : http://journals.openedition.org/paleo/1894

DOI : $10.4000 /$ paleo.1894

ISSN : 2101-0420

Éditeur

SAMRA

Édition imprimée

Pagination : 49-62

ISSN : $1145-3370$

Référence électronique

Vincent Mourre, Marc Jarry, David Colonge et Laure-Amélie Lelouvier, "Le débitage sur enclume aux Bosses (Lamagdelaine, Lot, France) », PALEO [En ligne], Numéro spécial | 2009-2010, mis en ligne le 23 avril 2012, consulté le 07 juillet 2020. URL : http://journals.openedition.org/paleo/1894 ; DOI : https:// doi.org/10.4000/paleo.1894

\section{cc) (i) $\Theta$}

PALEO est mis à disposition selon les termes de la licence Creative Commons Attribution - Pas d'Utilisation Commerciale - Pas de Modification 4.0 International 


\title{
LE DÉBITAGE SUR ENCLUME AUX BOSSES (Lamagdelaine, Lot, France)
}

\author{
Vincent MOURRE(1), Marc JARRY(2), David COLONGE ${ }^{(3)}$, \\ et Laure-Amélie LELOUVIER(4)
}

\begin{abstract}
Résumé : Le site des Bosses correspond à une vaste occupation de plein air du Paléolithique moyen ancien. II a livré une cinquantaine de nucléus issus d'un débitage sur enclume aux dépens de galets de quartz et de quartzite. Le débitage sur enclume peut être considéré ici comme une méthode de taille à part entière, permettant la production récurrente d'éclats faiblement prédéterminés. Il est délicat d'isoler au cas par cas les éclats issus de cette méthode mais une approche statistique des angles d'éclatements permet de confirmer que percussion sur enclume et percussion classique d'un bloc tenu en main ont été utilisés sur le site.
\end{abstract}

Mots-clés : Paléolithique moyen ancien, OIS 9, débitage sur enclume, quartz, quartzite.

Abstract: Bipolar-on-anvil at " Les Bosses » (Lamagdelaine, Lot, France). Les Bosses is a large Early Middle Paleolithic open air site. It yielded about fifty cores resulting from bipolar on anvil flaking of quartz and quartzite pebbles. Bipolar on anvil flaking appears to be here a real flaking method leading to the recurrent production of slightly predetermined flakes. It is difficult to determine for each single flake if it was detached using this method but a statistical study of the flaking angles confirms that bipolar on anvil flaking and classical flaking of a hand-held block were used on the site.

Key-words: Early Middle Paleolithic, OIS 9, bipolar-on-anvil flaking, quartz, quartzite.

\section{PRÉCISIONS TERMINOLOGIQUES PRÉLIMINAIRES}

Si la percussion sur enclume est avant tout une technique, au sens de " moyen élémentaire d'action sur la matière ", cette technique est aussi utilisée dans le cadre du débitage sur enclume, que nous qualifierons de méthode à part entière, au sens de " succession raisonnée de gestes, destinée à atteindre un objectif fixé » (d'après Inizan et al. 1995 et Pelegrin 1995).

Malgré de nombreuses tentatives de mise au point (Bordes 1947 ; Balout 1967 ; Tixier et al. 1980 ; Inizan et al. 1995), une certaine confusion terminologique persiste autour des techniques utilisant les enclumes et les percuteurs dormants. Le terme enclume est employé en technologie lithique par analogie avec la "masse de fer aciéré, montée sur un billot, sur laquelle on forge les métaux " (Petit Robert, 1992) : il s'agit donc, au sens propre, d'un instrument servant de support à un objet sur lequel des coups sont portés à l'aide d'un troisième élément. Une enclume sera donc un percuteur immobile utilisé comme support, et jouant un rôle direct ou indirect, mais jamais exclusif, dans un processus de taille (débitage, façonnage ou retouche). II y a donc nécessairement trois éléments : l'enclume elle-

(1) INRAP et TRACES/UTAH UMR 5608 du CNRS - Les Haut Arthèmes, 84560 Ménerbes - vincent.mourre@inrap.fr (2) INRAP et TRACES/UTAH UMR 5608 du CNRS - 13 rue du négoce, ZA des Champs Pinsons,

31650 Saint-Orens-de-Gameville - marc.jarry@inrap.fr

(3) INRAP et TRACES/UTAH UMR 5608 du CNRS - Dardenne, 46300 Le Vigan - david.colonge@inrap.fr

(2) INRAP et TRACES/UTAH UMR 5608 du CNRS - 13 rue du négoce, ZA des Champs Pinsons,

31650 Saint-Orens-de-Gameville - laure-amelie.lelouvier@inrap.fr 
même, la pièce taillée et un percuteur (fig. 1). Le rôle de l'enclume est direct dans les cas où la force appliquée par le percuteur et la réaction de l'enclume se situent dans un même axe : on parlera alors de « taille axiale sur enclume ». À l'inverse, le rôle de l'enclume est indirect dans les cas où la force appliquée par le percuteur et la réaction de l'enclume ne se situent pas dans le même axe : on parlera alors de " taille non axiale sur enclume ". L'emploi d'enclumes est relativement classique tout au long du Paléolithique lorsqu'il s'agit notamment d'ouvrir des plans de frappe aux dépens d'un nodule de silex ovoïde ou sphérique. II intervient aussi dans le cadre de débitages d'éclats aux dépens d'autres matériaux.

Bien que l'expression « percuteur dormant » ait été proposée par F. Bordes comme synonyme d'enclume (Bordes 1961, p. 13), nous pensons qu'il est justifié de limiter son emploi aux cas où une pièce à tailler est frappée sur un percuteur fixe posé au sol, ou tout au moins immobile. Un percuteur dormant est donc un percuteur dur immobile sur lequel est frappée la pièce à tailler. Celle-ci est alors le seul vecteur de l'énergie nécessaire au détachement d'un éclat (Mourre et Colonge, ce volume).

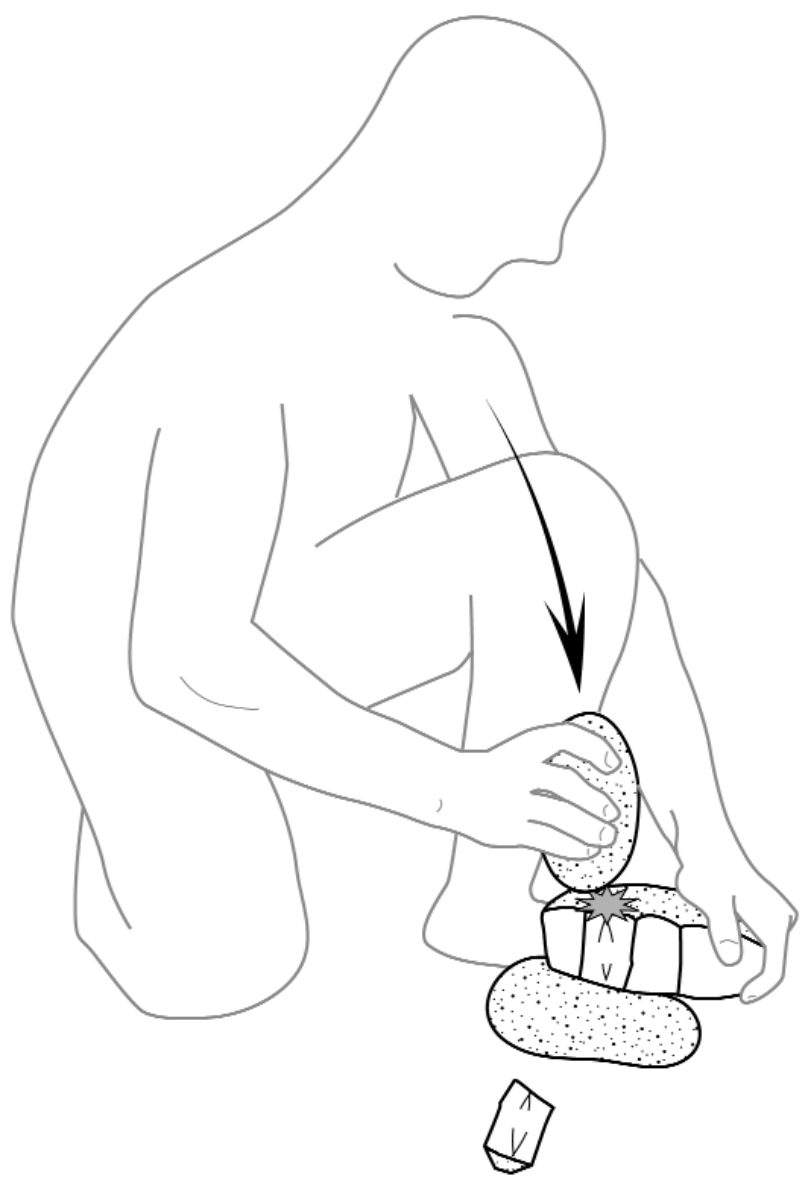

Figure 1 - Débitage sur enclume.

Figure 1 - Bipolar-on-anvil flaking.

\section{LA MÉTHODE DE DÉBITAGE SUR ENCLUME}

\section{Bref historique}

L'emploi de la percussion sur enclume a été reconnu anciennement par $\mathrm{H}$. Breuil au sein de l'industrie en quartz de Zhoukoudian (anciennement transcrit Chou-Kou-Tien ou Choukoutien) en Chine. Sous le nom de «taille bipolaire ", il donne, avec R. Lantier, une description particulièrement précise pour l'époque du déroulement du débitage et des stigmates obtenus. II considère toutefois cette méthode comme "laborieuse » pour le débitage du quartz : « II faut frapper fort et longtemps avant d'obtenir autre chose qu'une poussière de quartz, en même temps qu'un écrasement des deux extrémités du galet percuté. (...) Ce procédé ne permet pas d'obtenir de grands éclats, mais il présente l'avantage, là où les autres méthodes de taille sont inopérantes, de débiter entièrement un bloc de quartz, la taille manuelle ou sur enclume produisant des polyèdres subsphériques sur lesquels la percussion ordinaire n'a plus de prise » (Breuil et Lantier 1951, p. 71-72). La présence de débitage sur enclume à Zhoukoudian est confirmée par les travaux récents (Gao 2000).

François Bordes décrit lui aussi la "percussion "écrasée" ou percussion sur enclume » et note : "Il est difficile par ce procédé de savoir d'avance quel éclat on obtiendra, et il n'est guère intéressant que dans le cas de matières très dures, telles que le quartz » (Bordes 1947, p. 16).

Bien qu'elle ait été reconnue de longue date par des préhistoriens de renom, force est de constater que la technique du débitage sur enclume n'a pas bonne presse auprès d'eux, puisqu'ils évoquent des situations " dramatiques » (Breuil), et estiment que son utilisation n'autorise aucun contrôle des éclats débités ni aucune prédétermination (Bordes). Nous considérons qu'il s'agit là de généralisations abusives, basées sur des cas particuliers ou des tests limités.

\section{Principes physiques mis en œuvre}

Plus récemment, J. Pelegrin évoque la « percussion posée sur enclume (dite alors bipolaire), parfois seul moyen d'entamer ou fractionner des blocs ou galets sphéroïdes, comme de «fendre» par leur tranche des plaques d'une matière litée. Le mécanisme de la fracture (en «split» pour les anglo-saxons) est alors différent de la fracture conchoïdale, et se marque de stigmates particuliers, analogues à ceux que portent les pièces esquillées, car, comme pour ces dernières, le choc est porté dans l'axe de la pièce et non pas obliquement » (Pelegrin 1995, p. 21).

Comme l'a démontré $\mathrm{H}$. Bertouille dans ses Théories physiques et mathématiques de la taille des outils préhistoriques, les mécanismes mis en œuvre lors de la percussion d'une pièce dont une extrémité est fixe (percussion sur enclume) sont différents de ceux intervenant lors de la percussion de deux pièces dont les deux extrémités sont libres (nucléus tenu dans une main, frappé par un percuteur tenu dans l'autre). II ressort de son étude que la per- 
cussion sur enclume présente de nombreux avantages sur la percussion classique : il apparaît notamment que l'onde de compression se réfléchit sur l'enclume sans se modifier. Les contraintes qu'elle provoque s'ajoutent encore après l'impact, jusqu'à la fracturation éventuelle, et par conséquent « la force à transmettre au nucléus pour obtenir des résultats identiques peut, à cause des additions successives, être moindre dans le cas d'un nucléus fixe que celle à appliquer à un nucléus libre » (Bertouille 1989, pp. 20-21).

\section{Schéma conceptuel du débitage sur enclume du sud-ouest de la France}

Le débitage sur enclume relève d'une conception originale du débitage, même si un de ses principaux éléments de définition est l'emploi d'une technique particulière : la percussion directe au percuteur dur d'un bloc posé sur une enclume. Cette conception existe de manière indépendante au moins dans le cas du sud-ouest de la France où elle a été observée dans différents sites (Mourre 2004). Elle n'est directement comparable à aucune des autres conceptions du débitage connues au Paléolithique inférieur et moyen (Levallois, Discoïde, Kombewa, Quina, SSDA...).

Le nucléus comporte une surface de plan de frappe opposée à une surface de contrecoup (fig. 2). Le plus souvent, toutes deux sont néocorticales. Ces deux surfaces peu- vent être sub-parallèles, mais elles peuvent aussi converger ou diverger vers la surface de débitage (fig. 3). Dans chacun des cas, les produits auront des caractéristiques morpho-métriques différentes, en particulier en ce qui concerne l'angle d'éclatement. II semblerait que les rôles des deux surfaces puissent être inversés au cours d'une séquence de débitage, même si aucun élément autre qu'expérimental ne peut appuyer cette idée pour l'instant.

La surface de plan de frappe est percutée tandis que la surface de contrecoup est en contact avec l'enclume : une fracturation se produit entre ses deux points de contact, conduisant à la production d'un éclat dont les caractéristiques peuvent être prévues plus ou moins facilement en fonction de la matière première ainsi que du niveau de compétence et d'expérience du tailleur. Les produits recherchés présentent des caractéristiques morphotechniques déterminées par les enlèvements antérieurs : ils sont donc prédéterminés au sens premier de ce terme. Ces éclats présentent le plus souvent deux tranchants sub-parallèles, à talon et bord distal cortical, voire à talon seul cortical. II est important de souligner que tous les produits ne présentent pas des stigmates d'impacts aux deux extrémités de leur face inférieure. Cette face inférieure peut être très plane (fracture en «split» évoquée précédemment sous la plume de J. Pelegrin) mais aussi présenter un conchoïde de percussion tout à fait équivalent à ceux obtenus par percussion classique d'un bloc tenu en main ; la

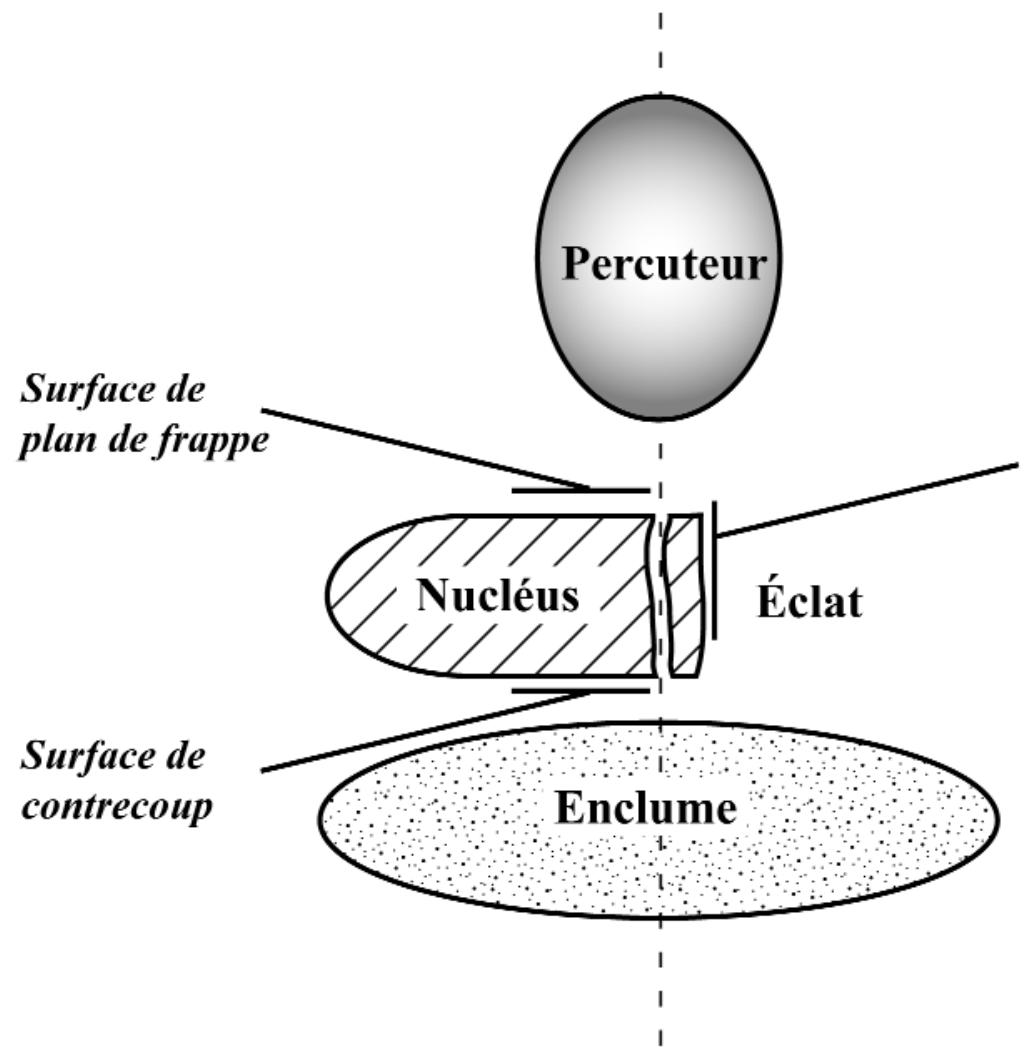

Surface de débitage
Figure 2 - Débitage sur enclume ; définition des éléments présents.

Figure 2 - Bipolar-on-anvil flaking; definition of the involved elements. 


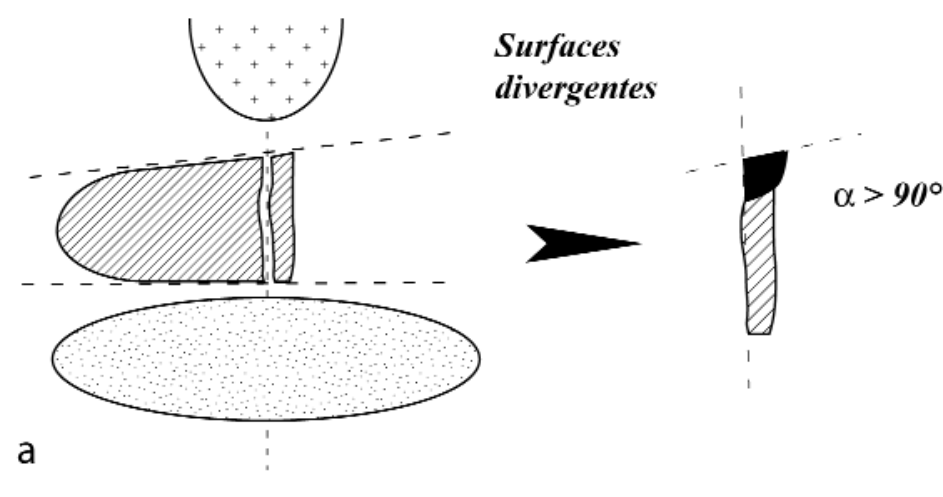

Figure 3 - Variabilité de l'angle d'éclatement des éclats en fonction des orientations respectives de la surface de plan de frappe et de la surface de débitage.

Figure 3 - Flaking angles variability of the flakes according to the respective orientation of the striking platform surface and the counterblow surface.
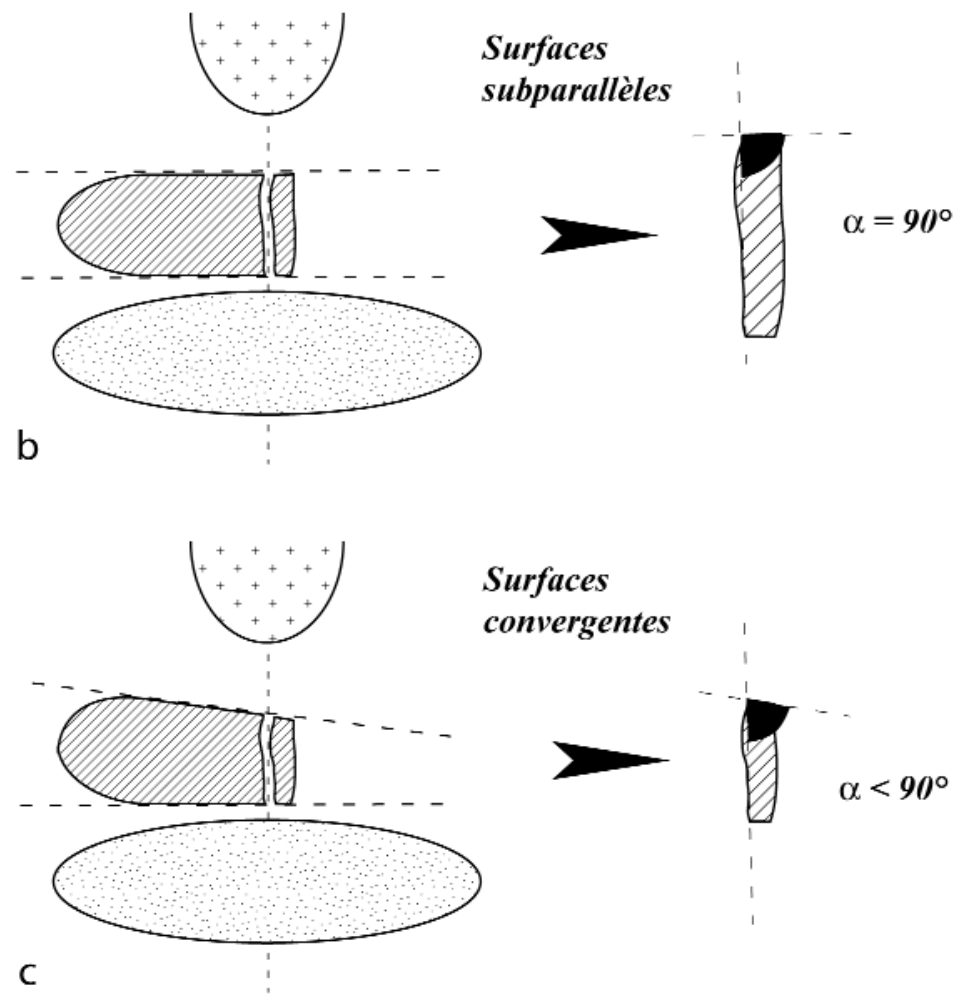

trajectoire de l'impact variant graduellement de parfaitement verticale et rectiligne à sensiblement oblique et tangentielle, toutes les morphologies intermédiaires sont également observées, rendant quasiment impossible la détermination pièce par pièce de la méthode à l'origine de la production des éclats lorsque le débitage sur enclume coexiste avec d'autres méthodes plus classiques au sein d'une série archéologique.

Le débitage sur enclume permet d'obtenir d'importantes séries récurrentes d'éclats prédéterminés aux dépens de blocs de matière première sur lesquels le débitage en maintien classique n'a pas de prise : galets sphériques ou dépourvus de dièdres corticaux, blocs parallélépipédiques épais, nucléus parvenus à une impasse technique par épuisement des angles favorables... La rentabilité de cette méthode est relativement importante, en ce sens qu'elle permet de produire des éclats fonctionnels de façon récurrente au prix d'un investissement technique minime. En effet aucune préparation ou configuration du nucléus n'est nécessaire, ce dernier présentant les caractéristiques requises dès sa sélection. Tout au plus est-il parfois nécessaire de supprimer les corniches générées dans la partie proximale de la surface de débitage par les négatifs des enlèvements antérieurs : la technique du débitage sur enclume non axial peut alors être utilisée avec profit.

La gestion du volume est techniquement peu complexe puisqu'il suffit pour entretenir les convexités latérales de la surface de débitage de produire de façon plus ou moins régulière des éclats " débordants " à talon, dos et bord distal en cortex (éclats $n^{\circ} 4$, " en quartier d'orange ", de A. Tavoso 1986). La notion de convexité distale n'a aucune validité ici puisqu'il est avantageux, dans le cadre du débi- 
tage axial sur enclume, que le point d'impact et le point de contrecoup soient à la verticale l'un de l'autre. Dans les limites des possibilités de préhension, le volume utile correspond au volume total du nucléus.

\section{LE SITE PALÉOLITHIQUE MOYEN ANCIEN DES BOSSES}

\section{Présentation générale}

Le gisement de plein air des Bosses se trouve à une douzaine de kilomètres à l'est de Cahors, sur la commune de Lamagdelaine (Lot, France) (fig. 4). II occupe la surface de la moyenne terrasse du Lot, près d'une confluence avec un vallon menant aux plateaux environnants, une position stratégique alliant disponibilité des matières premières, contrôle des voies de circulation naturelles du gibier et diversité écologique. En 2000, il a fait l'objet d'une importante opération de sauvetage urgent menée par l'AFAN. Cette opération a permis l'exploration du site sur plus de $6000 \mathrm{~m}^{2}$, dont près de 1000 fouillés manuellement. Le mobilier recueilli compte 2626 vestiges lithiques (Jarry et al. 2004, 2007).
Le niveau paléolithique s'insère dans une séquence stratigraphique explorée sur six mètres de puissance (fig. 5). II est localisé en surface d'un cône de déjection pléistocène déposé par un petit affluent du Lot dont les écoulements avaient cessé lors de l'occupation humaine. Les dépôts ont été préservés de l'érosion à la faveur de leur enfoncement dans une formation crypto-karstique constituée par une doline qui se développe dans le substrat calcaire sous la couverture alluviale, puis de leur scellement par des colluvions limoneuses. L'analyse micromorphologique d'un paléosol développé au sein de ces colluvions indique une histoire complexe marquée par l'association de figures pédologiques caractéristiques de phases interglaciaires et glaciaires : le niveau archéologique s'inscrivant dans ce paléosol, l'occupation du site serait antérieure au stade isotopique 7 (Bertran in Jarry et al. 2007).

Ces indications sont confortées par huit dates obtenues par thermoluminescence sur silex chauffé : si l'on exclut deux résultats aberrants et un âge sans doute trop jeune correspondant à une pièce à double patine, les valeurs sont proches de 300 ka BP (Debenham in Jarry et al. 2007).

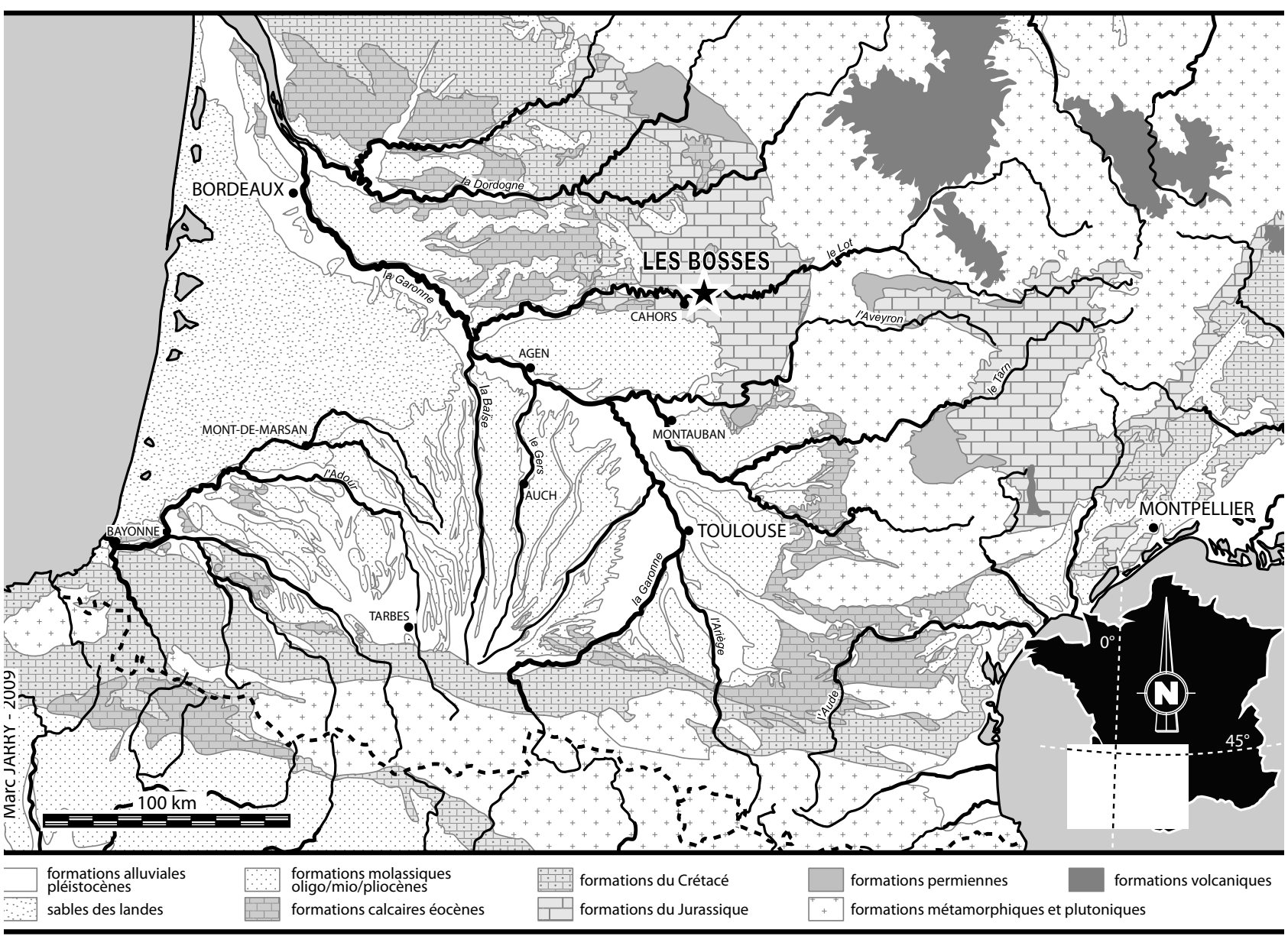

Figure 4 - Localisation du site des Bosses (Lamagdelaine, Lot) sur fond géologique simplifié.

Figure 4 - Location of Les Bosses site (Lamagdelaine, Lot, Southern France) on a simplified background. 


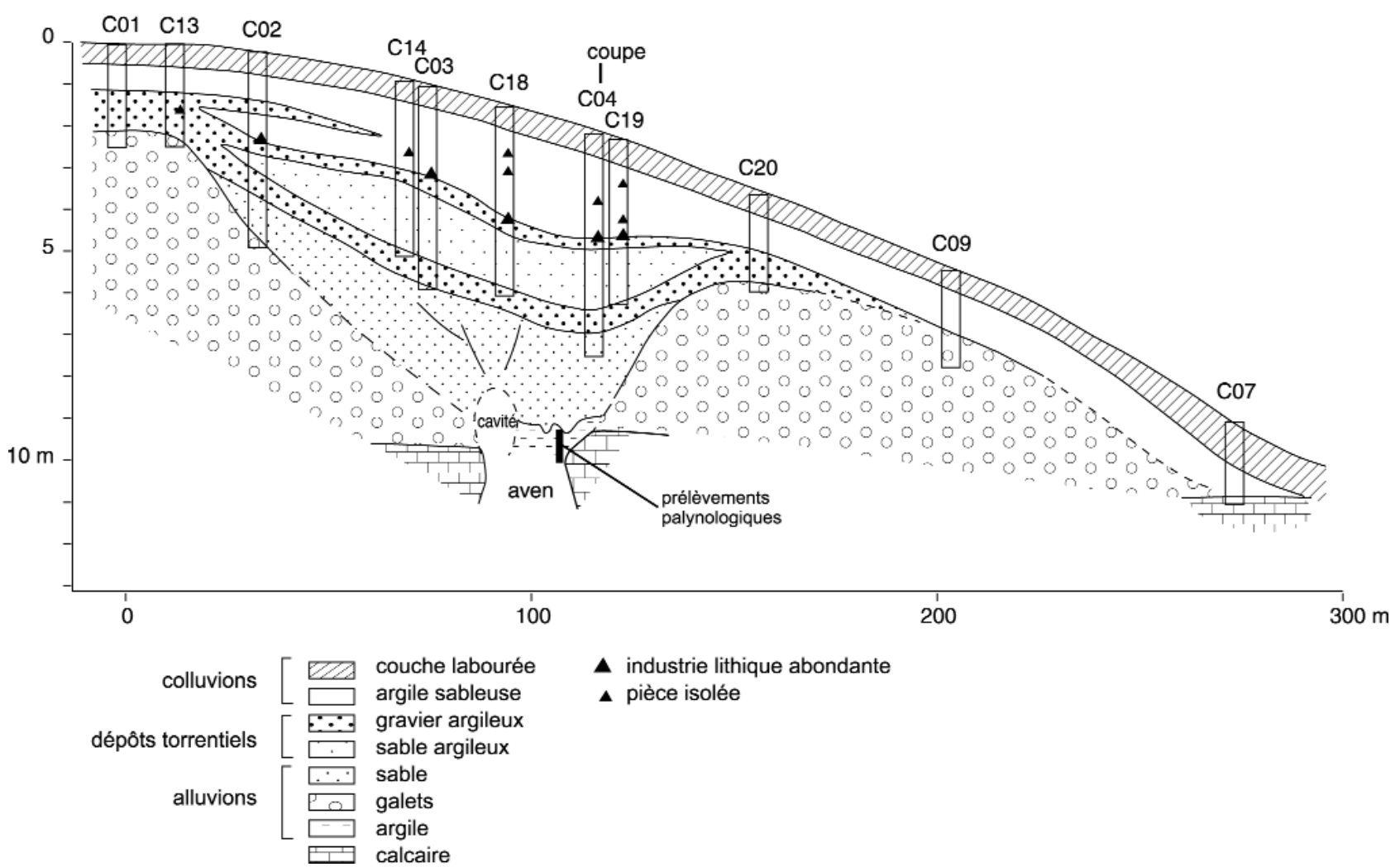

Figure 5 - Stratigraphie schématique du site (dessin P. Bertran, Inrap, d'après Jarry et al. 2007).

Figure 5 - Schematic stratigraphy of the site (drawing by P. Bertran, Inrap, after Jarry et al. 2007).

\section{Principales caractéristiques de l'industrie lithique}

La série lithique semble relativement homogène et n'a pas subi de perturbations post-dépositionnelles majeures. Elle est largement dominée par le groupe des quartz et des quartzites ( $83 \% ; n=2174)$, alors que les silex forment environ $15,5 \%(n=406)$ de l'ensemble et que les 1,5\% $(n=46)$ restant correspondent à des matériaux divers, le plus souvent magmatiques ou métamorphiques. Les matériaux utilisés sont très majoritairement issus des formations alluviales locales mais l'analyse pétroarchéologique révèle la présence de quelques silex évoquant des sources distantes d'une trentaine de kilomètres (Chalard et Turq in Jarry et al. 2007).

Une certaine économie des matières premières est perceptible, notamment pour les matériaux autres que quartz/quartzites et silex : ceux-ci ont fréquemment été utilisés pour réaliser des outils lourds sur galets. Ils ont apparemment été confectionnés hors de la zone fouillée, voire hors du site. Cette économie est également marquée dans le reste de la production, très nettement orientée vers le débitage d'éclats (fig. 6). Le débitage Discoïde est dominant, que ce soit sur quartz/quartzite (fig. 7) ou sur silex. D'autres schémas de production sont mis en œuvre, tels que le débitage Levallois (silex) (fig. 8) et le débitage sur enclume (quartz/quartzite) (fig. 9 et 10). Enfin, quelques pièces bifaciales $(n=14)$, souvent fragmentaires, attestent la mise en œuvre ponctuelle de schémas de façonnage. L'outillage retouché est assez bien représenté $(6,3 \%)$ mais est peu diversifié typologiquement (encoches, denticulés, becs) et la retouche est souvent irrégulière et/ou partielle.

La présence de débitage Levallois sur silex, la faible standardisation de l'outillage retouché et la rareté des pièces bifaciales permettent de rattacher l'industrie des Bosses à une phase ancienne du Paléolithique moyen. II est envisageable que les ressources minérales locales aient pu limiter l'expression de la connotation acheuléenne de cette série, plus marquée dans certaines industries sub-contemporaines attribuées à l'Acheuléen supérieur.

\section{LE DÉBITAGE SUR ENCLUME AUX BOSSES}

La série des Bosses comporte 53 nucléus rapportés au débitage sur enclume. Ils sont tous en quartz $(n=39)$ ou en quartzite $(n=14)$. Comme dans le reste du sud-ouest de la France, l'exploitation de silex par débitage sur enclume n'est pas documentée ici, bien qu'elle soit envisageable et attestée archéologiquement notamment au sein des industries pontiniennes (Laj Pannochia 1950 ; Grimaldi 1989 ; Bietti et al. ce volume). 

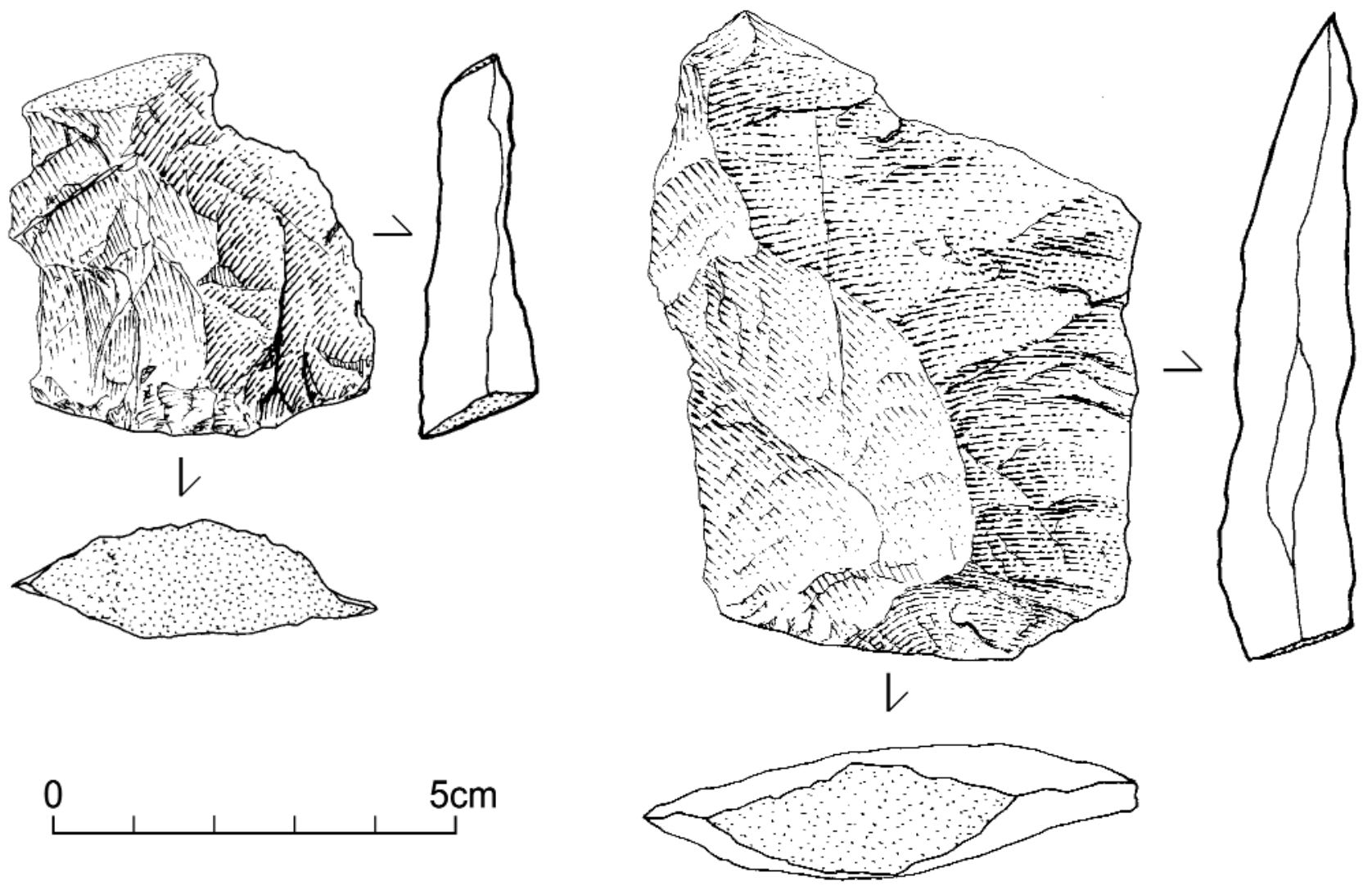

Figure 6 - Éclats à talons néocorticaux représentatifs de l'objectif principal de la production lithique des Bosses, quartz (à gauche) et quartzite (à droite).

Figure 6 - Quartz (left) and quartzite (right) flakes with neocortical butts typical of the main objective of lithic production in Les Bosses site.

Les principales caractéristiques métriques des nucléus débités sur enclume des Bosses sont présentées dans le tableau ci-dessous (tabl. 1). Ces valeurs sont très proches d'un matériau à l'autre.

\begin{tabular}{|l|c|c|c|c|}
\hline Quartz (n=39) & $\begin{array}{c}\text { longueur } \\
(\mathbf{m m})\end{array}$ & $\begin{array}{c}\text { largeur } \\
(\mathbf{m m})\end{array}$ & $\begin{array}{c}\text { épaisseur } \\
(\mathbf{m m})\end{array}$ & $\begin{array}{c}\text { masse } \\
(\mathbf{g})\end{array}$ \\
\hline moyenne & 71,87 & 60,26 & 48,44 & 367,64 \\
\hline écart type & 18,88 & 23,91 & 14,04 & 348,83 \\
\hline minimum & 42 & 24 & 23 & 70 \\
\hline maximum & 114 & 129 & 85 & 1270 \\
\hline
\end{tabular}

\begin{tabular}{|l|c|c|c|c|}
\hline Quartzite (n= 14) \\
\hline & $\begin{array}{c}\text { longueur } \\
(\mathbf{m m})\end{array}$ & $\begin{array}{c}\text { largeur } \\
(\mathbf{m m})\end{array}$ & $\begin{array}{c}\text { épaisseur } \\
(\mathbf{m m})\end{array}$ & $\begin{array}{c}\text { masse } \\
(\mathbf{g})\end{array}$ \\
\hline moyenne & 71,85 & 58,38 & 45,46 & 339,46 \\
\hline écart type & 21,12 & 26,71 & 14,13 & 429,35 \\
\hline minimum & 44 & 30 & 25 & 36 \\
\hline maximum & 129 & 127 & 79 & 1675 \\
\hline
\end{tabular}

Tableau 1 - Dimensions des nucléus issus du débitage sur enclume.

Table 1 - Dimensions of the cores reduced through bipolar on anvil flaking.

Les nucléus issus du débitage sur enclume présentent une structure volumétrique relativement caractéristique :
- ils comportent une surface de plan de frappe et une surface de contrecoup correspondant à deux faces plus ou moins planes du bloc originel ;

- ces deux surfaces sont séparées par une surface de débitage qui s'étend généralement sur une partie seulement de la périphérie du galet (morphologie de « chopper à front abrupt » de la typologie classique), plus rarement sur tout son pourtour (morphologie de « nucléus prismatique »).

Les difficultés d'identification de la méthode à partir des seuls éclats ont été évoquées précédemment (absence ou présence d'un bulbe sur la face inférieure, présence non systématique de stigmates d'impact au point de contrecoup, variabilité importante de l'angle d'éclatement, fracturation transversale du produit donnant deux pseudo-fragments proximaux, etc.) à tel point qu'il paraît illusoire dans le contexte considéré ici de tenter de rattacher individuellement tel éclat à telle ou telle méthode de débitage. Afin toutefois de confirmer que le débitage sur enclume avait bien joué le rôle important au sein de la production que laissait présager l'examen des nucléus, nous avons cherché un élément de preuve objectif et facilement observable sur les éclats. Cet élément est fourni par la mesure de l'angle d'éclatement des éclats (angle entre le talon et la face inférieure). En effet, l'une des caractéristiques de la percus- 

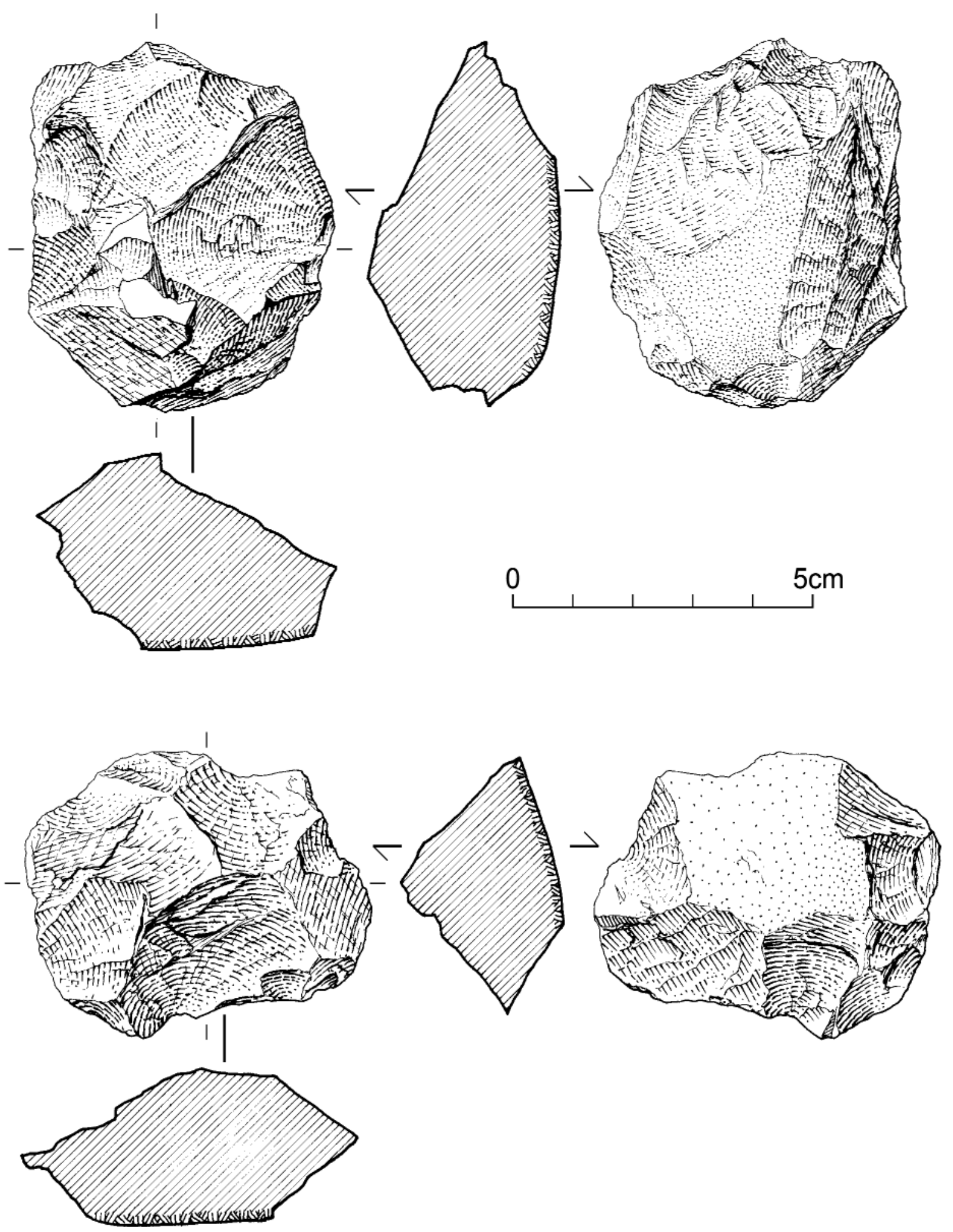

Figure 7 - Nucléus Discoïdes bifaciaux des Bosses, quartzite (en haut) et quartz (en bas).

Figure 7 - Quartzite (up) and quartz (down) bifacial Discoid cores from Les Bosses site. 

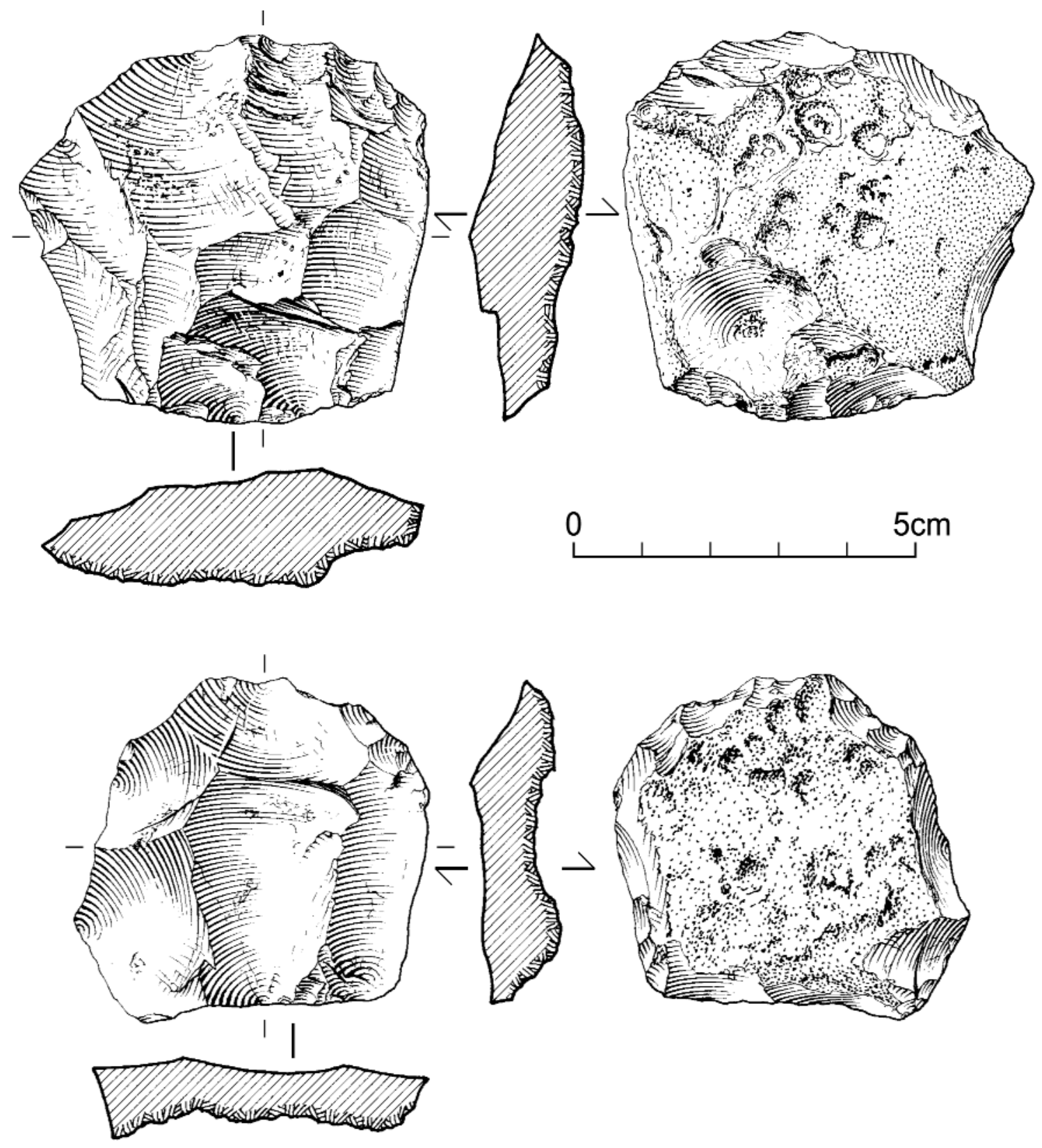

Figure 8 - Nucléus Levallois en silex des Bosses.

Figure 8 - Flint Levallois cores from Les Bosses site. 

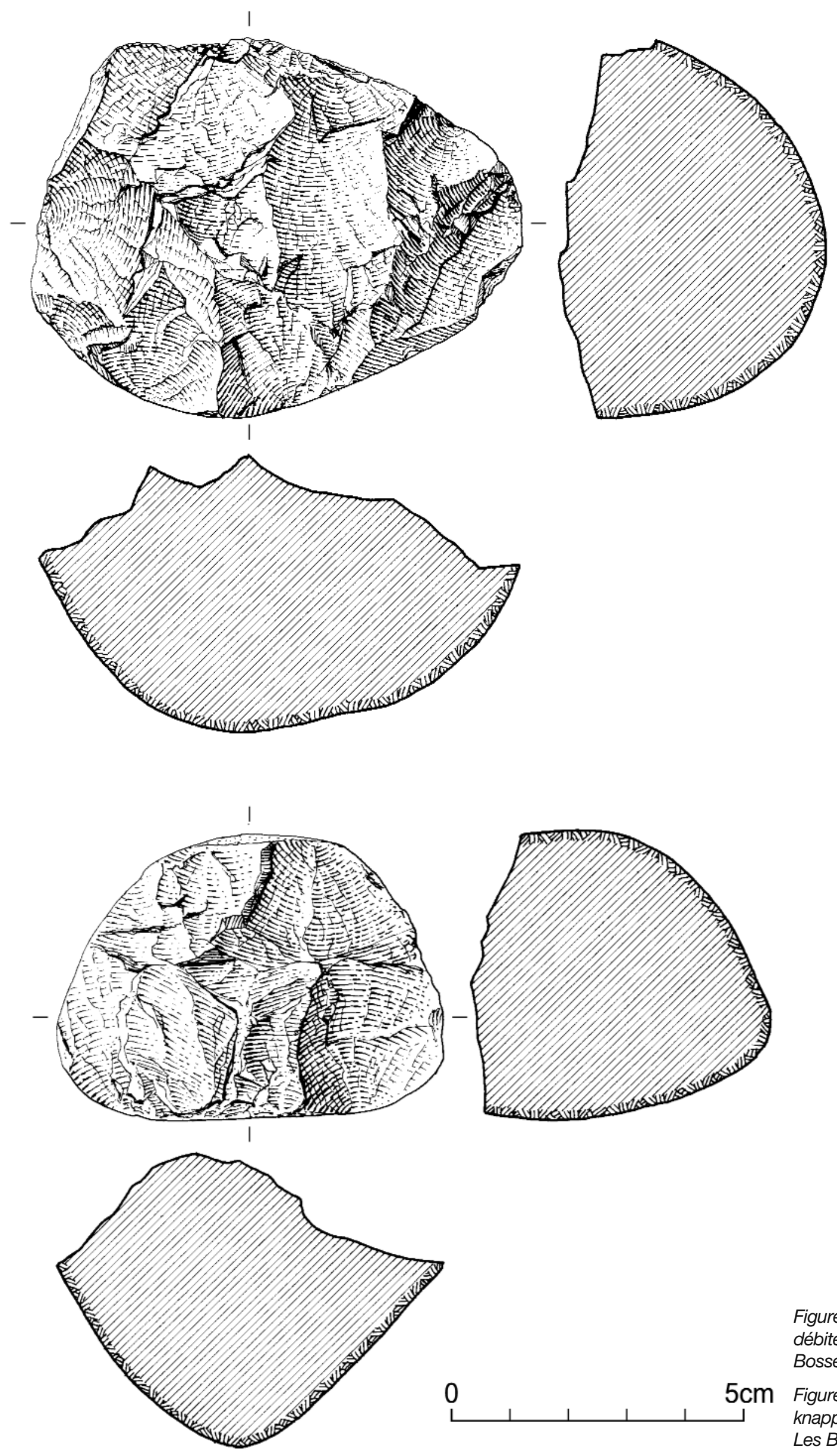

Figure 9 - Nucléus débités sur enclume des Bosses, quartz.

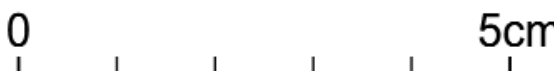

$5 \mathrm{~cm}$ Figure 9 - Quartz cores knapped on anvil from Les Bosses. 


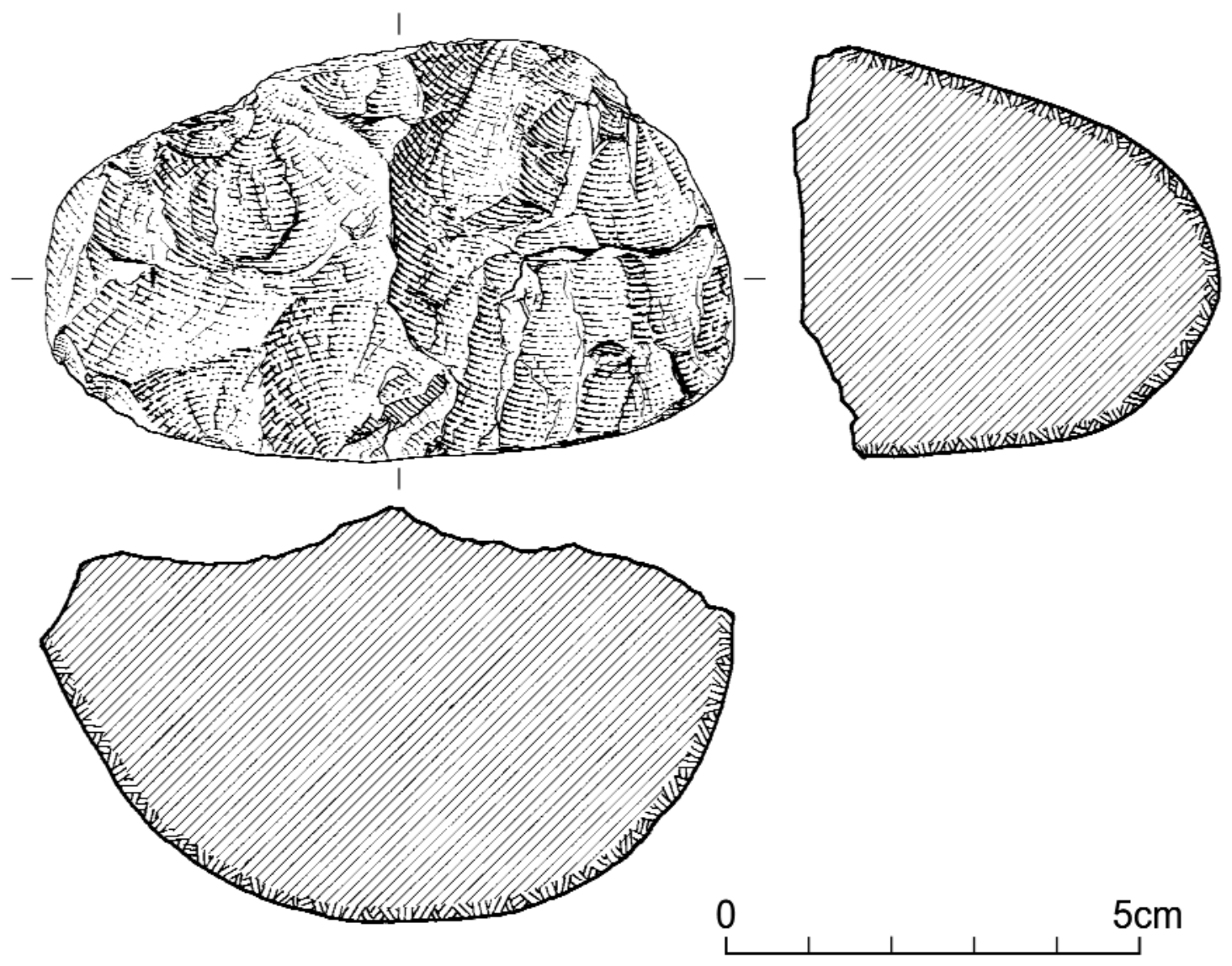

Figure 10 - Nucléus débité sur enclume des Bosses, quartzite.

Figure 10 - Quartzite core knapped on anvil from Les Bosses.

sion sur enclume est la possibilité de produire des éclats dont l'angle d'éclatement est inférieur à $90^{\circ}$. II convient de préciser qu'il ne s'agit pas d'un trait systématique, mais bien d'une possibilité liée à la morphologie des nucléus : suivant si les surfaces de plan de frappe et de contrecoup convergent ou non vers la surface de débitage, les angles d'éclatement seront plus ou moins fermés (Mourre 1996, 2004).

L'angle d'éclatement a été mesuré sur un échantillon de 171 éclats de quartz et de quartzite des Bosses. La répartition par classe de $5^{\circ}$ présente un profil bimodal dont les deux pics se situent respectivement autour de $90^{\circ}$ et de $110^{\circ}$ (fig. 11a). II est particulièrement intéressant de noter que le premier correspond au pic de répartition modale des angles d'un ensemble expérimental d'éclats débités sur enclume (fig. 11b). Il convient évidemment de relativiser la portée de ce résultat pour les raisons évoquées ci-dessus : les angles des éclats peuvent varier de façon conséquente d'un nucléus à l'autre et les données expérimentales utilisées correspondent au regroupement de quatre séries de produits dont les modes respectifs s'échelonnent de 80 à $100^{\circ}$ (fig. 11c). Nos résultats expérimentaux concernant le débitage sur enclume sont sensiblement équivalents à ceux publiés par H. Kobayashi (1975) (fig. 11d). Le second pic proche de $110^{\circ}$ semble correspondre au pic de répartition modale des angles d'éclatement obtenus expérimentalement par percussion directe au percuteur d'un bloc tenu en main (fig. 11e). Nos résultats sont à rapprocher des résultats expérimentaux obtenus par J.-M. Geneste pour le débitage Levallois sur silex, donnant un angle moyen de $100,0^{\circ}$ pour les produits Levallois, de $102,3^{\circ}$ pour les produits corticaux et de $108,5^{\circ}$ pour les éclats de préparation de plans de frappe (Geneste 1985, pp. 262-264). II est donc tentant de voir dans la répartition des angles de détachement des éclats de quartz et de quartzite des Bosses le cumul de deux populations issues pour l'une du débitage sur enclume et pour l'autre du débitage par percussion directe classique. Les rôles respectifs de ces deux techniques ne peuvent cependant pas être évalués avec précision à partir des seuls éclats, compte tenu du chevauchement des deux populations. 

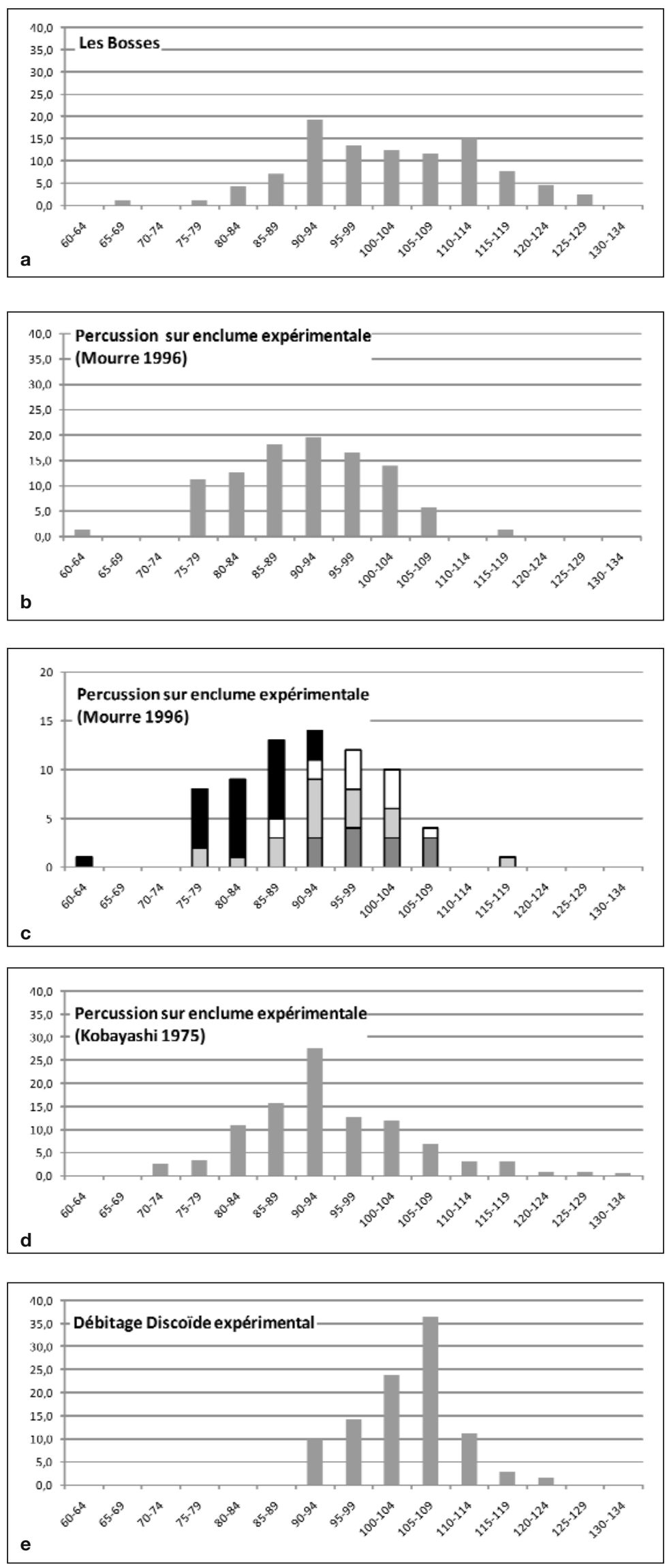

Figure 11 - Angles d'éclatements de séries d'éclats par classes de $5^{\circ}$; a. : éclats des Bosses, b. : éclats expérimentaux débités sur enclumes, c. : mêmes éclats expérimentaux débités sur enclume mais en distinguant chaque série expérimentale, $d$ 'éclats expérimentaux débités sur enclumes d'après Kobayashi 1975, e. : éclats expérimentaux produits par débitage Discoïde d'un nucléus tenu en main $(a, b$, $d$ et e en pourcentage, $c$ en effectif).

Figure 11 - Flaking angles of different flakes series (classes of $5^{\circ}$ ); a.: Les

Bosses flakes, b.: bipolar on anvil experimental flakes, c.: same bipolar on anvil experimental flakes but distinguishing the different experimental series, d.: bipolar on anvil experimental flakes according to Kobayashi 1975, e.: experimental flakes produced through Discoid flaking of a handheld core $(a, b, d$ and e expressed in percentage, $c$ in real number). 


\section{CONCLUSIONS}

Plus qu'une simple technique utilisée de façon anecdotique lors de la production d'industries archaïques, le débitage sur enclume peut être considéré, dans le Paléolithique inférieur et moyen du Sud-Ouest de la France, comme une méthode de débitage à part entière. Cette méthode permet d'obtenir des séries récurrentes significatives d'éclats présentant un certain degré de prédétermination, aux dépens de matières premières ou de formes de blocs difficilement exploitables par percussion directe en maintien classique. Dans l'aire géographique considérée, elle a été essentiellement documentée sur galets de quartz et quartzite, jamais sur silex (Colonge et Mourre 2009). Les éclats produits présentent une importante variabilité morpho-technique et ne comportent pas toujours de stigmates diagnostiques. La reconnaissance de la méthode passe donc le plus souvent par l'analyse des nucléus.

Aux Bosses, le débitage sur enclume semble relever d'un choix technique réalisé en amont de la chaîne opératoire, dès la sélection des blocs, pour produire une part non négligeable des éclats. C'est le cas également pour une partie des nucléus débités sur enclume mis au jour dans la couche 4 du gisement voisin de Coudoulous (Jaubert et Mourre 1996 ; Jaubert et al. 2005). Dans ce même site, le débitage sur enclume semble également être une solution technique adoptée lorsque survient une impasse technique (accumulation des réfléchissements, absence d'angles favorables, etc.) dans le cadre d'un débitage Discoïde unifacial. Dans d'autres cas, comme à Mauran et peut-être à La Borde, le débitage sur enclume pourrait être utilisé plus ponctuellement pour produire des éclats différents de ceux de la production principale (Jaubert et Mourre 1996 ; Mourre 2004).

\section{BIBLIOGRAPHIE}

BALOUT L. 1967 - Procédés d'analyse et questions de terminologie dans l'étude des ensembles industriels du Paléolithique inférieur en Afrique du Nord. In: Bishop, W. W. et Clark, J. D. (dir.), Background to evolution in Africa, Chicago, p. 701-735.

BERTOUILLE H. 1989 - Théories physiques et mathématiques de la taille des outils préhistoriques. Cahiers du Quaternaire $n^{\circ} 15$, CNRS éd., $100 \mathrm{p}$

BORDES F. 1947 - Étude comparative des différentes techniques de taille du silex et des roches dures. L'Anthropologie, t. 51, p. 1-29.

BORDES F. 1961 - Typologie du Paléolithique ancien et moyen. Bordeaux: Delmas, Publications de l'Institut de Préhistoire de l'Université de Bordeaux, Mémoire $\mathrm{n}^{\circ} 1$, $111 \mathrm{p}$.

BORDES F. 1967 - Considérations sur la typologie et les techniques dans le Paléolithique. Quartär, 18, p. 25-55.
BREUIL H. et LANTIER R. 1951 - Les Hommes de la pierre ancienne - Paléolithique et Mésolithque. Paris: Payot, $335 \mathrm{p}$.

COLONGE D. et MOURRE V. 2009 - Quartzite et quartzites : aspects pétrographiques, économiques et technologiques des matériaux majoritaires du Paléolithique ancien et moyen du Sud-Ouest de la France. In: Grimaldi, S. et Cura, S. (dir.), Technological analysis on quartzite exploitation, XVème Congrès de I'UISPP, 4-9 septembre 2006, Lisbonne, vol. 39, session WS 15, BAR International Series 1998, p. 3-12.

GAO X. 2000 - Core reduction at Zhoukoudian locality 15. Archaeology, ethnology \& anthropology of Eurasia, $n^{\circ} 3,3$, p. 2-12.

GENESTE J.-M. 1985 - Analyse lithique d'industries moustériennes du Périgord : une approche technologique du comportement des groupes humains au Paléolithique moyen. Thèse de Doctorat. Bordeaux: Université de Bordeaux I, $567 \mathrm{p}$.

GRIMALDI S. 1989 - Aprroccio tecnologico all'industria litica pontiniana di grotta Breuil - monte Circeo, Latina - tramite la sperimentazione. Roma: Università « La sapienza », Facoltà di Lettere e Filosofia.

INIZAN M.-L., REDURON-BALLINGER M., ROCHE H. et TIXIER J. 1995 - Préhistoire de la Pierre Taillée - $t .4$ : Technologie de la pierre taillée. Meudon: CREP, 199 p.

JARRY M., BERTRAN P., COLONGE D., LELOUVIER L.-A. et MOURRE V. 2004 - Le gisement paléolithique moyen ancien des Bosses à Lamagdelaine (Lot, France). In: Van Peer, P., Bonjean, D. et Semal, P. (dir.), Session 5 : Le Paléolithique moyen - Sessions générales et posters, BAR S1239 - Actes du XIV'̀me Congrès de I'UISPP, Liège, 28 sept. 2001, p. 177-185.

JARRY M., COLONGE D., LELOUVIER L.-A. et MOURRE V. (dir.) 2007 - Les Bosses 1, Lamagdelaine (Lot) : un gisement paléolithique moyen antérieur à l'avant-dernier Interglaciaire sur la moyenne terrasse du Lot. Société Préhistorique Française, Travaux 7, 158 p.

JAUBERT J., KERVAZO B., BRUGAL J.-P., CHALARD P., FALGUĖRES C., JARRY M., JEANNET M., LEMORINI C., LOUCHART A., MAKSUD F., MOURRE V., QUINIF Y. et THIÉBAUT C. 2005 - Coudoulous I (Tour-de-Faure, Lot), site du Pléistocène moyen en Quercy. Bilan pluridisciplinaire. In: Molines, N., Moncel, M.-H. et Monnier, J.-L. (dir.), Données récentes sur les modalités de peuplement et sur le cadre chronostratigraphique, géologique et paléogéographique des industries du Paléolithique inférieur et moyen en Europe, BAR International Series 1364, Actes du Colloque International de Rennes, 22-25 septembre 2003, p. 227-251.

JAUBERT J. et MOURRE V. 1996 - Coudoulous, Le Rescoundudou, Mauran : diversité des matières premières et variabilité des schémas de production d'éclats. In: Bietti, 
A. et Grimaldi, S. (dir.), Proceedings of the International Round Table : Reduction processes (" chaines opératoires ») for the European Mousterian, Rome: Quaternaria Nova Vl, p. 313-341.

KOBAYASHI H. 1975 - The experimental study of bipolar flakes. In: Swanson, E. (dir.), Lithic Technology - Making and using stone tools, The Hague: Mouton, p. 115-128.

LAJ PANNOCCHIA F. 1950 - L'industria pontiniana della grotta di S. Agostino (Gaeta). Rivista di Scienze Preistoriche, vol. V, fasc. 1-4, p. 67-86.

MOURRE V. 1996 - Le débitage sur enclume au Paléolithique inférieur et moyen. Techniques, méthodes et schémas conceptuels. Article de DEA. Université de Paris $X$ - Nanterre, 45 p.

MOURRE V. 2004 - Le débitage sur enclume au Paléolithique moyen dans le Sud-Ouest de la France. In: Van Peer, P., Bonjean, D. et Semal, P. (dir.), Session 5 : Paléolithique moyen, BAR S1239 - Actes du XIVème Congrès de I'UISPP, Liège, 2-8 sept. 2001, p. 29-38.
PELEGRIN J. 1995 - Technologie lithique : le Châtelperronien de Roc-de-Combe (Lot) et de La Côte (Dordogne). Paris: CNRS Editions, Cahiers du Quaternaire $\mathrm{n}^{\circ} 20,297$.

TAVOSO A. 1986 - Le Paléolithique inférieur et moyen du Haut-Languedoc. Gisements des terrasses alluviales du Tarn, du Dadou, de l'Agout, du Sor et du Fresquel. Université de Provence, Ed. du Laboratoire de Paléontologie Humaine et de Préhistoire, Études Quaternaires, 5, (1978), $404 \mathrm{p}$.

TIXIER J. 1980 - Préhistoire - La taille expérimentale des roches dures. In: Encyclopedia Universalis, p. 1199-1201. 